\title{
Polyharmonic Multiquadric Particular Solutions for Reissner/Mindlin Plate
}

\author{
Chia-Cheng Tsai ${ }^{1,2}$ \\ ${ }^{1}$ Department of Marine Environmental Engineering, National Kaohsiung Marine University, Kaohsiung 81157, Taiwan \\ ${ }^{2}$ International Wave Dynamics Research Center, National Cheng Kung University, Tainan 70955, Taiwan
}

Correspondence should be addressed to Chia-Cheng Tsai; tsaichiacheng@gmail.com

Received 18 September 2014; Accepted 29 December 2014

Academic Editor: Jaromir Horacek

Copyright (C) 2015 Chia-Cheng Tsai. This is an open access article distributed under the Creative Commons Attribution License, which permits unrestricted use, distribution, and reproduction in any medium, provided the original work is properly cited.

\begin{abstract}
Analytical particular solutions of the polyharmonic multiquadrics are derived for both the Reissner and Mindlin thick-plate models in a unified formulation. In the derivation, the three coupled second-order partial differential equations are converted into a product operator of biharmonic and Helmholtz operators using the Hörmander operator decomposition technique. Then a method is introduced to eliminate the Helmholtz operator, which enables the utilization of the polyharmonic multiquadrics. Then, the analytical particular solutions of displacements, shear forces, and bending or twisting moments corresponding to the polyharmonic multiquadrics are all explicitly derived. Numerical examples are carried out to validate these particular solutions. The results obtained by the present method are more accurate than those by the traditional multiquadrics and splines.
\end{abstract}

\section{Introduction}

Boundary-type numerical methods have been emerged as a popular research field because only boundary discretizations are required when they are applied to solve homogeneous problems. These methods include the boundary element method (BEM) [1], method of fundamental solutions (MFS) $[2,3]$, and Trefftz methods (TM) $[4,5]$. When they are applied for solving an inhomogeneous partial differential equation, the dual reciprocity method (DRM) can be used $[6,7]$.

In the early development of DRM, the ad hoc radial function, $1+r$, was exclusively used. In order to improve the accuracy of the computation, Golberg and Chen [8] and Karur and Ramachandran [9] applied the theory of radial basis functions (RBFs) to the DRM. Among these RBFs, Hardy's multiquadrics (MQ) [10] and Duchon's augmented polyharmonic spline (APS) [11] are the most popular ones. For example, Golberg [12, 13], Chen [14], and Karur and Ramachandran [9] demonstrated the superiority of the APS over the ad hoc radial function. Then, Golberg et al. [15] further improved the accuracy of the approximated particular solution by utilizing the MQ. Recently, Tsai [16] generalized the MQ to the polyharmonic multiquadrics (PMQ) and showed further accuracy improvement of the PMQ over the traditional MQ. In this study, the accuracy improvement of the PMQ over the MQ and APS is demonstrated for the Reissner and Mindlin thick-plate models.

When the MQ is adopted in a DRM procedure, the applicability depends on the availability of the analytical particular solution of the basis function associated with the partial differential operator of a given problem. Golberg et al. [15] derived the analytical particular solutions for the Laplace equation. Samaan and Rashed [17, 18] and Tsai and Hsu [19] found the analytical particular solutions, respectively, for the two- and three-dimensional elasticity problems, which can be converted to a biharmonic equation using the GalerkinPapkovich vector [20]. Basically, the applicability of MQ is limited to the harmonic and biharmonic operators [21] before Tsai [16] derived the analytical particular solutions of MQ associated with the polyharmonic operators, which are denoted as the PMQ in the following.

When applying the DRM for solving the Mindlin thickplate model [22], Wen et al. [23] and Tsai and $\mathrm{Wu}$ [24] found the analytical particular solutions for the ad hoc RBF and the APS, respectively. In addition, the analytical particular solutions for the Chebyshev polynomials [25] and the APS [26] were derived for the Reissner thick-plate model [27, 28]. Basically, a product operator of biharmonic and Helmholtz 


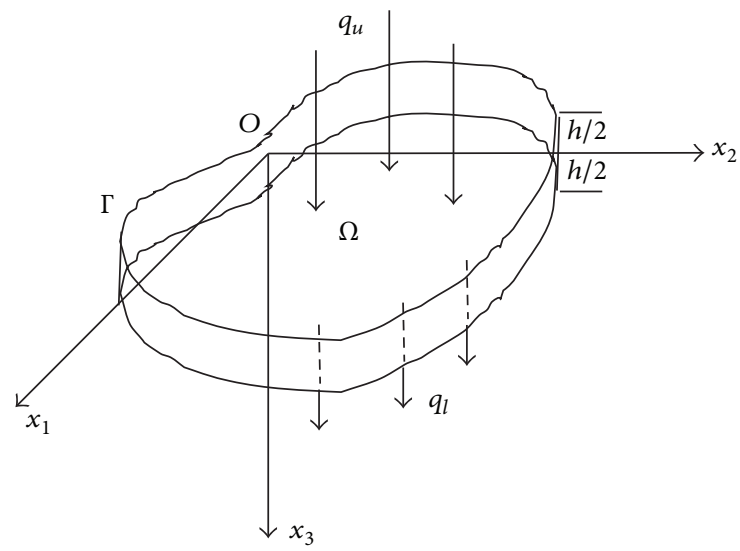

FIgURE 1: Configuration of a thick plate.

operators resulted when applying the Hörmander operator decomposition technique [29] to the coupled PDEs of the Reissner and Mindlin thick-plate models as demonstrated in the previous studies [24-26]. This seems to infer that the accuracy improvement of the PMQ over the APS is not possible for the thick-plate models.

In this study, a method is introduced to eliminate the Helmholtz operator, which makes the application of the PMQ for the thick-plate models possible. Numerical experiments are carried out to demonstrate the superior accuracy of the PMQ over the traditional MQ and the first few orders of the APS.

This paper is organized as follows: the unified Reissner/Mindlin plate model is introduced in Section 2. Then, a review of the PMQ is given in Section 3. And the particular solutions of the PMQ associated with the Reissner/Mindlin plate model are derived in Section 4. Section 5 gives the formulation of MFS-DRM. Finally, some numerical experiments are carried out to validate the particular solutions in Section 6 and the conclusions are drawn in Section 7.

\section{Reissner/Mindlin Plate Model}

In the following, indices $i, j, k$ are in the range $\{1,2,3\}$ and indices $\alpha, \beta, \gamma$ are in the range $\{1,2\}$. As described in Figure 1, we consider a plate of uniform thickness $h$ with its middle plane being a domain $\Omega$ with boundary $\Gamma$ in the $x_{1}-x_{2}$ plane and thickness coordinate $x_{3}$. The plate is subjected to a normal loading with intensity $q\left(x_{1}, x_{2}\right)$. Then, the equations of equilibrium are given by

$$
\begin{gathered}
\frac{\partial Q_{\alpha}}{\partial x_{\alpha}}+q=0, \\
\frac{\partial \Upsilon_{\alpha \beta}}{\partial x_{\beta}}-Q_{\alpha}=0,
\end{gathered}
$$

where $Q_{\alpha}$ is the transverse shear force and $\Upsilon_{\alpha \beta}$ is the bending or twisting moment.

In the Reissner [27, 28] and Mindlin [22] thick-plate theory, $u_{\alpha}$ represents an average slope angle and $u_{3}$ is the lateral deflection of the plate in the middle surface. According to the theories, the constitutive equations are given by

$$
\begin{gathered}
Q_{\alpha}=\frac{D(1-v) \lambda^{2}}{2}\left(u_{\alpha}+\frac{\partial u_{3}}{\partial x_{\alpha}}\right) \\
\Upsilon_{\alpha \beta}=D \frac{1-v}{2}\left(\frac{\partial u_{\alpha}}{\partial x_{\beta}}+\frac{\partial u_{\beta}}{\partial x_{\alpha}}+\frac{2 v}{1-\nu} \frac{\partial u_{\gamma}}{\partial x_{\gamma}} \delta_{\alpha \beta}\right) \\
+\frac{\varepsilon \nu}{(1-v) \lambda^{2}} q \delta_{\alpha \beta}
\end{gathered}
$$

with

$$
\begin{aligned}
\lambda & =\frac{\pi}{h}, \\
\varepsilon & =0
\end{aligned}
$$

for the Mindlin plate model and

$$
\begin{gathered}
\lambda=\frac{\sqrt{10}}{h}, \\
\varepsilon=1
\end{gathered}
$$

for the Reissner plate model. Combining (1) (2) results in the following governing equation:

$$
\widetilde{L}\left(\begin{array}{l}
u_{1} \\
u_{2} \\
u_{3}
\end{array}\right)=-\widetilde{E} q
$$

with

$$
\begin{gathered}
\widetilde{L}=\left(\begin{array}{ccc}
\frac{D(1-v)}{2}\left(\nabla^{2}-\lambda^{2}\right)+\frac{D(1+v)}{2} \frac{\partial^{2}}{\partial x_{1}{ }^{2}} & \frac{D(1+v)}{2} \frac{\partial^{2}}{\partial x_{1} \partial x_{2}} & -\frac{D \lambda^{2}(1-v)}{2} \frac{\partial}{\partial x_{1}} \\
\frac{D(1+v)}{2} \frac{\partial^{2}}{\partial x_{1} \partial x_{2}} & \frac{D(1-v)}{2}\left(\nabla^{2}-\lambda^{2}\right)+\frac{D(1+v)}{2} \frac{\partial^{2}}{\partial x_{2}{ }^{2}} & -\frac{D \lambda^{2}(1-v)}{2} \frac{\partial}{\partial x_{2}} \\
\frac{D \lambda^{2}(1-v)}{2} \frac{\partial}{\partial x_{1}} & -\frac{D \lambda^{2}(1-v)}{2} \frac{\partial}{\partial x_{2}} & \frac{D \lambda^{2}(1-v)}{2} \nabla^{2}
\end{array}\right) \\
\widetilde{E}=\left(\begin{array}{c}
\frac{\varepsilon v}{(1-v) \lambda^{2}} \frac{\partial}{\partial x_{1}} \\
\frac{\varepsilon v}{(1-v) \lambda^{2}} \frac{\partial}{\partial x_{2}}
\end{array}\right)
\end{gathered}
$$


where $D$ and $v$ are Young's modulus and Poisson ratio, respectively.

Equation (5) is a coupled system of three second-order PDEs for the three unknown functions $u_{j}$, and, therefore, three boundary conditions are required as

$$
\begin{aligned}
& \mathbf{B}_{1} \cdot \mathbf{u}=H_{1}\left(x_{1}, x_{2}\right), \\
& \mathbf{B}_{2} \cdot \mathbf{u}=H_{2}\left(x_{1}, x_{2}\right), \\
& \mathbf{B}_{3} \cdot \mathbf{u}=H_{3}\left(x_{1}, x_{2}\right),
\end{aligned}
$$

where $H_{i}\left(x_{1}, x_{2}\right)$ are given boundary data.

\section{Polyharmonic Multiquadrics}

Before deriving the particular solutions of PMQ associated with the Reissner/Mindlin plate model, we give a short review on the PMQ previously derived by the method of undetermined coefficients together with the Laurent series in [16]. Considering the $L$ th order PMQ $\psi_{L}$, it is governed by the following hierarchical relation:

$$
\begin{gathered}
\nabla^{2} \psi_{L}=\psi_{L-1}, \\
\psi_{0}=\sqrt{c^{2}+r^{2}}, \\
\left(\nabla^{2}\right)^{i} \psi_{L}=0 \quad \text { as } r \longrightarrow 0 \text { for } 0 \leq i \leq L-1 .
\end{gathered}
$$

Here, (10) is introduced so that the PMQ are hierarchically unique and $c$ is a shape parameter to tune the numerical accuracy [10].

Then, by using the method of undetermined coefficients, we can find the solution of (8) (10) as

$$
\begin{aligned}
\frac{\psi_{L}}{c^{2 L+1}}= & \sum_{j=0}^{L} A_{L, j} R^{2 j} \sqrt{1+R^{2}} \\
& +\sum_{j=0}^{L-1} B_{L, j} R^{2 j} \ln \left(\frac{1+\sqrt{1+R^{2}}}{2}\right)+\sum_{j=0}^{L-1} C_{L, j} R^{2 j}
\end{aligned}
$$

with

$$
R=\frac{r}{c}
$$

for any positive integer $L$. And, the unknown $C_{L, i}$ in (11) can be solved by using the uniqueness condition (10) as

$$
C_{L, i}=-\left(\sum_{j=0}^{i} P_{L, i, j}+\sum_{j=0}^{i-1} Q_{L, i, j}\right) \quad \text { for } 0 \leq i \leq L-1
$$

with

$$
P_{L, i, j}=\frac{(-1)^{i-j-1}(2 i-2 j-3) ! !}{(i-j) ! 2^{i-j}} A_{L, j},
$$

$$
Q_{L, i, j}=\frac{(-1)^{i-j+1}(2 i-2 j-1) ! !}{(i-j) ! 2^{i-j+1}(i-j)} B_{L, j}
$$

In (14), the double factorial is defined as

$$
\begin{aligned}
& n ! ! \\
& \quad \begin{cases}n \cdot(n-2) \cdots 5 \cdot 3 \cdot 1 & n \text { is an odd positive integer } \\
n \cdot(n-2) \cdots 6 \cdot 4 \cdot 2 & n \text { is an even positive integer } \\
1 & n=0,-1 \\
\frac{(-1)^{(n+1) / 2}}{(-n-2) ! !} & n \leq-3 \text { is an odd negative } \\
& \text { integer. }\end{cases}
\end{aligned}
$$

The PMQ defined in (11) can be expanded into the Maclaurin series as

$$
\frac{\psi_{L}}{c^{2 L+1}}=\sum_{i=L}^{\infty}\left(\sum_{j=0}^{L} P_{L, i, j}+\sum_{j=0}^{L-1} Q_{L, i, j}\right) R^{2 i}
$$

Observing (16), we can conclude that the proposed PMQ are infinitely differentiable since they only consist of evencoefficient polynomials $[16,30]$. Then, substituting (16) into (8) and (9) and performing some mathematical manipulations can result in

$$
\begin{aligned}
& \sum_{j=0}^{L} \frac{P_{L, i+L, j} 4^{L}(i+L) !^{2}}{i !^{2}}+\sum_{j=0}^{L-1} \frac{Q_{L, i+L, j} 4^{L}(i+L) !^{2}}{i !^{2}} \\
& \quad=\frac{(-1)^{i-1}(2 i-3) ! !}{i ! 2^{i}} \text { for all nonnegative integer } i,
\end{aligned}
$$

which can be used for solving the unknown coefficients $A_{L, j}$ and $B_{L, j}$ in (11) and (16). In practice, we simply enforce $i$ equal to $0,1, \ldots, 2 L$ in (17) which results in $2 L+1$ linear equations for solving the $2 L+1$ unknown coefficients $A_{L, j}$ and $B_{L, j}$ of the PMQ. After $A_{L, j}$ and $B_{L, j}$ are obtained, (13) can be used to have $C_{L, j}$. Then, the PMQ $\psi_{L}$ can be computed simply by (11). For the cases when $r$ is very small or equal to zero, one can alternatively use the Maclaurin series (16).

For the purpose of a later derivation of the particular solutions associated with the Reissner/Mindlin plate model, we need to find the formulas when the radial differential operator $(d / r d r)^{M}$ is applied onto (11) and (16). This can be done for arbitrary nonnegative integer $M$ since the proposed 
PMQ are infinitely differentiable. Then, the required formulas for (11) and (16) can be derived, respectively, as

$$
\begin{aligned}
\left(\frac{d}{r d r}\right)^{M} \frac{\psi_{L}}{c^{2 L-2 M+1}}= & \sum_{j=0}^{L} A_{L, j} V_{M, j}(R) \\
& +\sum_{j=0}^{L-1} B_{L, j} W_{M, j}(R)+\sum_{j=0}^{L-1} C_{L, j} U_{M, j}(R), \\
\left(\frac{d}{r d r}\right)^{M} \frac{\psi_{L}}{c^{2 L-2 M+1}}= & \sum_{i=L}^{\infty}\left(\sum_{j=0}^{L} P_{L, i, j}+\sum_{j=0}^{L-1} Q_{L, i, j}\right) U_{M, i}(R)
\end{aligned}
$$

with

$$
\begin{aligned}
& U_{M, j}(R) \\
& \equiv\left(\frac{d}{R d R}\right)^{M} R^{2 j} \\
& \quad= \begin{cases}\frac{(2 j) ! !}{(2 j-2 M) ! !} R^{2 j-2 M}, & \text { for } j \geq M \geq 0, \\
0, & \text { for } M>j \geq 0, \\
\frac{(-1)^{M}(-2 j+2 M-2) ! !}{(-2 j-2) ! !} R^{2 j-2 M}, & \text { for } j<0,\end{cases}
\end{aligned}
$$

$V_{M, j}(R)$

$$
\begin{aligned}
& \equiv\left(\frac{d}{R d R}\right)^{M}\left(R^{2 j} \sqrt{1+R^{2}}\right) \\
& =\sum_{k=0}^{M} \frac{k !(M-k) !}{M !} U_{M-k, j}(R)\left(\frac{d}{R d R}\right)^{k} \sqrt{1+R^{2}},
\end{aligned}
$$

$W_{M, j}(R)$

$$
\begin{aligned}
& \equiv\left(\frac{d}{R d R}\right)^{M}\left(R^{2 j} \ln \left(\frac{1+\sqrt{1+R^{2}}}{2}\right)\right) \\
& =\sum_{k=0}^{M} \frac{k !(M-k) !}{M !} U_{M-k, j}(R)\left(\frac{d}{R d R}\right)^{k} \ln \left(\frac{1+\sqrt{1+R^{2}}}{2}\right) .
\end{aligned}
$$

In deriving (18), we have used

$$
c^{2 M}\left(\frac{d}{r d r}\right)^{M}=\left(\frac{d}{R d R}\right)^{M}
$$

And, in deriving (20) and (21), we have used the product rule of differentiation as follows:

$$
\begin{aligned}
& \left(\frac{d}{R d R}\right)^{M}(f g) \\
& \quad=\sum_{k=0}^{M} \frac{k !(M-k) !}{M !}\left(\left(\frac{d}{R d R}\right)^{M-k} f\right)\left(\left(\frac{d}{R d R}\right)^{k} g\right)
\end{aligned}
$$

with $f$ and $g$ being two arbitrary radial functions. Finally, additional formulas are required to complete (20) and (21), respectively, as follows:

$$
\left(\frac{d}{R d R}\right)^{k} \sqrt{1+R^{2}}=\frac{(-1)^{k-1}(2 k-3) ! !}{{\sqrt{1+R^{2}}}^{2 k-1}},
$$

$$
\begin{aligned}
& \left(\frac{d}{R d R}\right)^{k} \ln \left(\frac{1+\sqrt{1+R^{2}}}{2}\right) \\
& = \begin{cases}\frac{1}{R^{2}}-\frac{1}{R^{2} \sqrt{1+R^{2}}}, & \text { for } k=1 \\
U_{k-1,-1}(R) & \\
-\sum_{l=0}^{k-1} \frac{l !(k-1-l) !}{(k-1) !} U_{k-1-l,-1}(R)\left(\frac{d}{R d R}\right)^{l} & \frac{1}{\sqrt{1+R^{2}}}, \\
& \text { for } k>1\end{cases}
\end{aligned}
$$

with

$$
\left(\frac{d}{R d R}\right)^{l} \frac{1}{\sqrt{1+R^{2}}}=\frac{(-1)^{l}(2 l-1) ! !}{{\sqrt{1+R^{2}}}^{2 l+1}} .
$$

The proposed formulas can be implemented into a floating-point subroutine for evaluating $(d / r d r)^{M} \psi_{L}(r)$ with arbitrary nonnegative integers $M$ and $L$. Therefore, the subroutine can be invoked when implementing numerical methods. In addition, the source codes can be obtained from the author via e-mails.

\section{Analytical Particular Solution of Plate Model}

Analytical particular solutions are required when the DRM is applied for solving a Reissner/Mindlin plate problem under an arbitrary loading. In this section, the analytical particular solutions of the PMQ associated with the Reissner/Mindlin plate model will be derived, which are governed by

$$
\widetilde{L}\left(\begin{array}{l}
u_{1} \\
u_{2} \\
u_{3}
\end{array}\right)=-\widetilde{E} \psi_{L}(r),
$$

where

$$
r=\sqrt{x_{1}^{2}+x_{2}^{2}}
$$

Formally, the particular solutions $u_{j}\left(x_{1}, x_{2}\right)$, governed by (27), should be obtainable by using the Hörmander 
operator decomposition technology [29], which begins with the definition of the adjoint operator $\widetilde{L}^{\text {adj }}$ as

$$
\widetilde{L}^{\mathrm{adj}} \widetilde{L}=\widetilde{L} \widetilde{L}^{\mathrm{adj}}=\operatorname{det}(\widetilde{L}) \widetilde{I}
$$

where $\operatorname{det}(\widetilde{L})$ is the determinant of $\widetilde{L}$ and $\widetilde{I}$ is the threeby-three identity matrix. Here, both $\operatorname{det}(\widetilde{L})$ and $\widetilde{L}^{\text {adj }}$ can be obtained by linear algebraic manipulations, respectively, defined as

$$
\begin{aligned}
& \operatorname{det}(\widetilde{L})=\frac{D^{3} \lambda^{2}(1-\nu)^{2}}{4}\left(\nabla^{2}-\lambda^{2}\right) \nabla^{2} \nabla^{2}, \\
& \widetilde{L}^{\text {adj }}=\left(\begin{array}{ccc}
2 \nabla^{4}-\left[(1+\nu) \nabla^{2}+(1-\nu) \lambda^{2}\right] \frac{\partial^{2}}{\partial x_{1}{ }^{2}} & -\left[(1+\nu) \nabla^{2}+(1-\nu) \lambda^{2}\right] \frac{\partial^{2}}{\partial x_{1} \partial x_{2}} & (1-\nu) \frac{\partial}{\partial x_{1}}\left(\nabla^{2}-\lambda^{2}\right) \\
-\left[(1+\nu) \nabla^{2}+(1-\nu) \lambda^{2}\right] \frac{\partial^{2}}{\partial x_{1} \partial x_{2}} & 2 \nabla^{4}-\left[(1+\nu) \nabla^{2}+(1-\nu) \lambda^{2}\right] \frac{\partial^{2}}{\partial x_{2}^{2}} & (1-\nu) \frac{\partial}{\partial x_{2}}\left(\nabla^{2}-\lambda^{2}\right) \\
-(1-\nu) \frac{\partial}{\partial x_{1}}\left(\nabla^{2}-\lambda^{2}\right) & -(1-\nu) \frac{\partial}{\partial x_{2}}\left(\nabla^{2}-\lambda^{2}\right) & \frac{\left(\nabla^{2}-\lambda^{2}\right)\left[2 \nabla^{2}-(1-\nu) \lambda^{2}\right]}{\lambda^{2}}
\end{array}\right) .
\end{aligned}
$$

Then, the particular solutions can be found by assuming

$$
\left(\begin{array}{l}
u_{1}\left(x_{1}, x_{2}\right) \\
u_{2}\left(x_{1}, x_{2}\right) \\
u_{3}\left(x_{1}, x_{2}\right)
\end{array}\right)=\widetilde{L}^{\mathrm{adj}} \widetilde{E} F(r),
$$

where $F(r)$ is an unknown function to be determined. In (32), the required operator $\widetilde{L}^{\text {adj }} \widetilde{E}$ can be obtained as

$$
\begin{aligned}
\widetilde{L}^{\text {adj }} \widetilde{E} & \left(\begin{array}{c}
\frac{D^{2}(1-\nu)}{4} \frac{\partial}{\partial x_{1}}\left(\nabla^{2}-\lambda^{2}\right)\left[\varepsilon \nu \nabla^{2}+(1-\nu) \lambda^{2}\right] \\
\frac{D^{2}(1-\nu)}{4} \frac{\partial}{\partial x_{2}}\left(\nabla^{2}-\lambda^{2}\right)\left[\varepsilon \nu \nabla^{2}+(1-\nu) \lambda^{2}\right] \\
\frac{D^{2}(1-\nu)}{4}\left(\nabla^{2}-\lambda^{2}\right)\left[(2-\varepsilon \nu) \nabla^{2}-(1-\nu) \lambda^{2}\right]
\end{array}\right) .
\end{aligned}
$$

After substituting (32) into (27) and using (29) and (30), the resulted equation indicates that the following particular solution is required:

$$
-\frac{D^{3} \lambda^{2}(1-\nu)^{2}}{4}\left(\nabla^{2}-\lambda^{2}\right) \nabla^{2} \nabla^{2} F=\psi_{L} .
$$

However, the analytical particular solution of the above equation is not known due to the existence of the Helmholtz operator.

In this study, we find that the Helmholtz operator can be eliminated by rewriting (33) as

$$
\widetilde{L}^{\text {adj }} \widetilde{E}=\frac{D^{3} \lambda^{2}(1-\nu)^{2}}{4}\left(\nabla^{2}-\lambda^{2}\right) \widetilde{E}_{1},
$$

where the operator $\widetilde{E}_{1}$ is defined by

$$
\widetilde{E}_{1}=\frac{1}{D \lambda^{2}(1-v)}\left(\begin{array}{c}
\frac{\partial}{\partial x_{1}}\left[\varepsilon \nu \nabla^{2}+(1-v) \lambda^{2}\right] \\
\frac{\partial}{\partial x_{2}}\left[\varepsilon \nu \nabla^{2}+(1-v) \lambda^{2}\right] \\
(2-\varepsilon v) \nabla^{2}-(1-v) \lambda^{2}
\end{array}\right)
$$

Then, (29) and (35) can be combined to have

$$
\begin{aligned}
\widetilde{L} \widetilde{L}^{\text {adj }} \widetilde{E} & =-\frac{D^{3} \lambda^{2}(1-\nu)^{2}}{4}\left(\nabla^{2}-\lambda^{2}\right) \nabla^{2} \nabla^{2} \widetilde{E} \\
& \Longrightarrow \widetilde{L}\left\{\frac{D^{3} \lambda^{2}(1-\nu)^{2}}{4}\left(\nabla^{2}-\lambda^{2}\right)\right\} \widetilde{E}_{1} \\
& =-\frac{D^{3} \lambda^{2}(1-\nu)^{2}}{4}\left(\nabla^{2}-\lambda^{2}\right) \nabla^{2} \nabla^{2} \widetilde{E} \\
& \Longrightarrow \widetilde{L} \widetilde{E}_{1}=-\nabla^{2} \nabla^{2} \widetilde{E} .
\end{aligned}
$$

The above equations have suggested us to modify the Hörmander operator decomposition technology by assuming

$$
\left(\begin{array}{l}
u_{1}\left(x_{1}, x_{2}\right) \\
u_{2}\left(x_{1}, x_{2}\right) \\
u_{3}\left(x_{1}, x_{2}\right)
\end{array}\right)=\widetilde{E}_{1} F_{1}(r)
$$

where $F_{1}(r)$ is an unknown function to be determined. Substituting (38) into (27) and using (37) result in

$$
\nabla^{2} \nabla^{2} F_{1}(r)=\psi_{L}
$$

By using the PMQ definitions (8) and (9), we can have the particular solution of (39) as

$$
F_{1}(r)=\psi_{L+2} .
$$

Substituting (40) into (38) and using (36) can result in the desired analytical particular solutions of displacements as

$$
\begin{aligned}
& u_{\alpha}\left(x_{1}, x_{2}\right) \\
& =\frac{-x_{\alpha}}{D \lambda^{2}(1-v)} \\
& \quad \cdot\left(\varepsilon v\left(\frac{d}{r d r}\right) \psi_{L+1}+(1-\nu) \lambda^{2}\left(\frac{d}{r d r}\right) \psi_{L+2}\right), \\
& u_{3}\left(x_{1}, x_{2}\right)=\frac{-(2-\varepsilon \nu) \psi_{L+1}+(1-\nu) \lambda^{2} \psi_{L+2}}{D \lambda^{2}(1-\nu)},
\end{aligned}
$$


where we have used

$$
\nabla^{2} F_{1}=\nabla^{2} \psi_{L+2}=\psi_{L+1}
$$

In addition, the required formulas of $d \psi_{L+1} / r d r$ and $d \psi_{L+2} /$ $r d r$ have been provided in the previous section. Then, the corresponding shear forces and bending/twisting moments can be obtained by using the definitions

$$
\begin{gathered}
Q_{\alpha}\left(x_{1}, x_{2}\right)=\frac{D(1-v) \lambda^{2}}{2}\left(u_{\alpha}^{M}+\frac{\partial u_{3}^{M}}{\partial x_{\alpha}}\right) \\
\Upsilon_{\alpha \beta}\left(x_{1}, x_{2}\right)=\frac{D(1-v)}{2}\left(\frac{\partial u_{\alpha}^{M}}{\partial x_{\beta}}+\frac{\partial u_{\beta}^{M}}{\partial x_{\alpha}}+\frac{2 \nu}{1-\nu} \frac{\partial u_{\gamma}^{M}}{\partial x_{\gamma}} \delta_{\alpha \beta}\right) \\
+\frac{\delta_{\alpha \beta} \varepsilon v \sqrt{r^{2}+c^{2}}}{\lambda^{2}(1-v)} .
\end{gathered}
$$

Equations (41), (42), (44), and (45) are sufficient to obtain the analytical particular solutions of shear forces and bending/twisting moments, respectively, as follows:

$$
\begin{gathered}
Q_{\alpha}\left(x_{1}, x_{2}\right)=-x_{\alpha}\left(\frac{d}{r d r}\right) \psi_{L+1}, \\
\Upsilon_{\alpha \beta}\left(x_{1}, x_{2}\right) \\
=-\delta_{\alpha \beta}\left\{\frac{\left(\varepsilon\left(\nu^{2}-v\right) \sqrt{r^{2}+c^{2}}+\nu(1-\nu) \lambda^{2} F_{2}(r)\right)}{\lambda^{2}(1-v)}\right. \\
+\frac{1}{\lambda^{2}}\left(\varepsilon \nu\left(\frac{d}{r d r}\right) \psi_{L+1}\right. \\
\left.+\frac{x_{\alpha} x_{\beta}}{\lambda^{2}}\left(\varepsilon \nu\left(\frac{d}{r d r}\right)^{2} \psi_{L+1}+(1-\nu) \lambda^{2}\left(\frac{d}{r d r}\right) \psi_{L+2}\right)\right\}
\end{gathered}
$$

where the additional terms $(d / r d r)^{2} \psi_{L+1}$ and $(d / r d r)^{2} \psi_{L+2}$ have been given in the previous section.

This completes the derivation of the analytical particular solutions of the PMQ associated with the Reissner/Mindlin plate model.

\section{MFS-DRM Formulation}

Now, we are in a position to review the application of the MFS-DRM procedure $[24,26]$ for solving the well-posed thick-plate problem governed by (5) and (7). First of all, we need the principle of superposition as

$$
u_{j}=u_{j}^{h}+u_{j}^{p}
$$

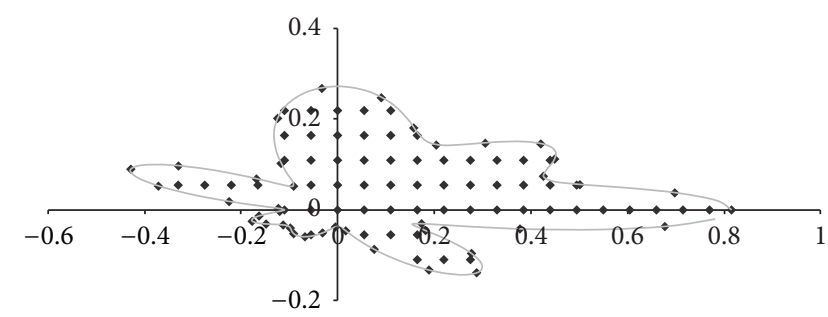

FIGURE 2: Field points of the DRM.

where the particular solution $u_{j}^{p}$ satisfies

$$
\widetilde{L}\left(\begin{array}{l}
u_{1}{ }^{p} \\
u_{2}{ }^{p} \\
u_{3}{ }^{p}
\end{array}\right)=-\widetilde{E} q
$$

without specifying any boundary condition. In addition, the homogeneous solution $u_{j}{ }^{h}$ satisfies

$$
\widetilde{L}\left(\begin{array}{c}
u_{1}{ }^{h} \\
u_{2}{ }^{h} \\
u_{3}{ }^{h}
\end{array}\right)=\left(\begin{array}{l}
0 \\
0 \\
0
\end{array}\right)
$$

with modified boundary conditions

$$
\begin{aligned}
& \mathbf{B}_{1} \cdot \mathbf{u}^{h}=H_{1}\left(x_{1}, x_{2}\right)-\mathbf{B}_{1} \cdot \mathbf{u}^{p} \\
& \mathbf{B}_{2} \cdot \mathbf{u}^{h}=H_{2}\left(x_{1}, x_{2}\right)-\mathbf{B}_{2} \cdot \mathbf{u}^{p} \\
& \mathbf{B}_{3} \cdot \mathbf{u}^{h}=H_{3}\left(x_{1}, x_{2}\right)-\mathbf{B}_{3} \cdot \mathbf{u}^{p},
\end{aligned}
$$

where $\mathbf{u}^{h}=\left(u_{1}{ }^{h}, u_{2}{ }^{h}, u_{3}{ }^{h}\right)$ and $\mathbf{u}^{p}=\left(u_{1}{ }^{p}, u_{2}{ }^{p}, u_{3}{ }^{p}\right)$.

In order to apply the DRM for solving the particular solution $u_{j}{ }^{p}$, the loading intensity needs to be first approximated by the PMQ as follows:

$$
q\left(x_{1}, x_{2}\right) \cong \sum_{n=1}^{N} a_{n} \psi_{L}\left(r_{n}\right)
$$

where $r_{n}$ is the distance between $\left(x_{1}, x_{2}\right)$ and the $n$th DRM field point, $\left(\xi_{n 1}, \xi_{n 2}\right)$, as depicted in Figure 2. In order to solve the unknown coefficient $a_{n}$, (52) should be collocated on the $N$ DRM field points as

$$
q\left(\xi_{l 1}, \xi_{l 2}\right)=\sum_{n=1}^{N} a_{n} \psi_{L}\left(r_{l n}\right)
$$

for $l=1,2, \ldots, N$ and $r_{l n}$ is the distance between the $l$ th and $n$th DRM field points. Then, the corresponding particular solution can be approximated by

$$
u_{j}^{p} \cong \sum_{n=1}^{N} a_{n} u_{j}\left(r_{n}\right)
$$


with the particular solutions $u_{j}(r)$ given by (41) and (42). Also, the particular solutions of shear force and bending or twisting moment can be approximated, respectively, as

$$
\begin{gathered}
Q_{\alpha}{ }^{p} \cong \sum_{n=1}^{N} a_{n} Q_{\alpha}\left(r_{n}\right), \\
\Upsilon_{\alpha \beta}{ }^{p} \cong \sum_{n=1}^{N} \alpha_{n} \Upsilon_{\alpha \beta}\left(r_{n}\right) .
\end{gathered}
$$

In (55) and (56), the particular solutions $Q_{\alpha}$ and $\Upsilon_{\alpha \beta}$ are given in (46) and (47), respectively.
After the particular solutions are approximated by the DRM, the modified boundary conditions (51) become well defined. Therefore, it is ready to use the MFS for solving the homogeneous solution. Formally, the homogeneous solution can be approximated by

$$
u_{j}^{h} \cong \sum_{k=1}^{3} \sum_{m=1}^{M} b_{m}^{k} u_{j k}^{*}\left(x_{1}, x_{2} ; s_{m 1}, s_{m 2}\right)
$$

where $u_{j k}{ }^{*}\left(x_{1}, x_{2} ; s_{1}, s_{2}\right)$ are the fundamental solutions defined by

$$
\widetilde{L}\left(\begin{array}{ccc}
u_{11}{ }^{*} & u_{12}{ }^{*} & u_{13}{ }^{*} \\
u_{21}{ }^{*} & u_{22}{ }^{*} & u_{23}{ }^{*} \\
u_{31}{ }^{*} & u_{31}{ }^{*} & u_{33}{ }^{*}
\end{array}\right)=-\left(\begin{array}{ccc}
\delta\left(x_{1}-s_{1}, x_{2}-s_{2}\right) & 0 & 0 \\
0 & \delta\left(x_{1}-s_{1}, x_{2}-s_{2}\right) & 0 \\
0 & 0 & \delta\left(x_{1}-s_{1}, x_{2}-s_{2}\right)
\end{array}\right)
$$

and $\left(s_{m 1}, s_{m 2}\right)$ are $M$ source points outside the plate domain as described in Figure 3. In (57), the $3 M$ unknowns $b_{m}^{k}$ can be solved by collocating the modified boundary conditions (51) on $M$ boundary points. More details of the MFS and the explicit fundamental solutions can be found in $[24,26]$. After both the particular and homogeneous solutions are solved, the principle of superposition (48) can be applied for obtaining the desired solution.

\section{Numerical Results}

In order to validate the derived particular solutions, three numerical cases are considered. Typically, $D=\lambda=2$ and $\nu=$ 0.3 are selected in these numerical studies. Furthermore, the MFS is always arranged to be sufficient and accurate such that the numerical errors are majorly controlled by the DRM. The root mean square errors (RMSEs) of the numerical solutions are defined as

$$
\sqrt{\frac{\sum_{i=1}^{3} \sum_{l=1}^{\bar{L}}\left(u^{\mathrm{NUM}}{ }_{i, l}-u^{\mathrm{ANA}}{ }_{i, l}\right)^{2}}{3 \bar{L}}},
$$

where $u^{\mathrm{NUM}}{ }_{i, l}$ is the numerical solution obtained by the MFS-DRM at the $l$ th point of the $\bar{L}$ considered positions and $u^{\mathrm{ANA}}{ }_{i, l}$ is the corresponding analytical solution of the problem. Here, $\bar{L}$ will be chosen to be sufficiently large to ensure the utilization of the RMSEs.

In our numerical experiments, we consider the following Reissner/Mindlin plate problem:

$$
\widetilde{L}\left(\begin{array}{l}
u_{1} \\
u_{2} \\
u_{3}
\end{array}\right)=-D \widetilde{E}\left\{\sin x_{1}\left(x_{2}+\sinh x_{2}+x_{2} \sinh x_{2}\right)\right\} \text {. }
$$

This problem has been considered for both the Mindlin [24] and the Reissner [26] cases and their analytical particular solutions can be unified as

$$
\begin{aligned}
& u_{1}=\left(\operatorname { c o s } x _ { 1 } \left\{48 x_{2}\left[\lambda^{2}(\nu-1)+\varepsilon \nu\right]\right.\right. \\
& -6\left[\left(1+x_{2}\right)^{2} \lambda^{2}(\nu-1)\right. \\
& \left.+\left(1+2 x_{2}\left(2+x_{2}\right)\right) \varepsilon \nu\right] \cosh x_{2} \\
& +\left[\left(9+x_{2}\left(9+2 x_{2}\left(3+x_{2}\right)\right)\right) \lambda^{2}(\nu-1)\right. \\
& \left.\left.\left.+12\left(1+x_{2}\right) \varepsilon \nu\right] \sinh x_{2}\right\}\right) \\
& \cdot\left(48 \lambda^{2}(1-\nu)\right)^{-1} \\
& u_{2}=\left(\operatorname { s i n } x _ { 1 } \left\{48\left[\lambda^{2}-\left(\varepsilon+\lambda^{2}\right) \nu\right]\right.\right. \\
& +\left[\left(3+x_{2}\left(3-2 x_{2}\left(3+x_{2}\right)\right)\right)\right. \\
& \left.\cdot \lambda^{2}(\nu-1)+12\left(1+x_{2}\right) \varepsilon \nu\right] \cosh x_{2} \\
& +3\left[-\lambda^{2}(\nu-1)\right. \\
& \left.\left.\left.+2\left(2 x_{2}\left(2+x_{2}\right)-1\right) \varepsilon \nu\right] \sinh x_{2}\right\}\right) \\
& \cdot\left(48 \lambda^{2}(\nu-1)\right)^{-1} \\
& u_{3}=\left(\operatorname { s i n } x _ { 1 } \left\{48\left[2+\lambda^{2}(1-v)-\varepsilon v\right] x_{2}\right.\right. \\
& +6\left[\lambda^{2}(\nu-1)\left(1+x_{2}\right)^{2}\right.
\end{aligned}
$$




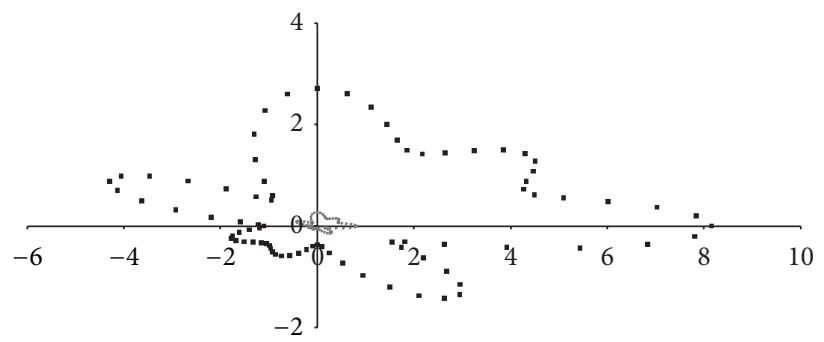

- Boundary points

- Source points

FIGURE 3: Boundary and source points of the MFS.

TABLE 1: The RMSEs for Example 1 (Div for divergence).

\begin{tabular}{lcccc}
\hline & $N=6 \times 6$ & $N=8 \times 8$ & $N=10 \times 10$ & $N=12 \times 12$ \\
\hline Chebyshev & $2.10 E-06$ & $8.62 E-09$ & $2.18 E-09$ & $3.81 E-10$ \\
MQ & $6.10 E-06$ & $4.30 E-07$ & $4.30 E-07$ & $2.74 E-08$ \\
PMQ $(L=2)$ & $6.04 E-06$ & $2.17 E-07$ & $2.05 E-08$ & $5.94 E-09$ \\
PMQ $(L=4)$ & $4.51 E-06$ & $1.20 E-07$ & $5.12 E-09$ & $1.66 E-09$ \\
APS (order $=1)$ & $2.22 E-04$ & $9.38 E-05$ & $4.96 E-05$ & $2.85 E-05$ \\
APS (order $=3)$ & $3.71 E-05$ & $9.08 E-06$ & $3.02 E-06$ & $1.49 E-06$ \\
APS (order $=5)$ & $4.88 E-06$ & $7.35 E-07$ & $1.90 E-07$ & $5.90 E-08$ \\
APS (order $=7)$ & Div & $8.14 E-08$ & $1.27 E-08$ & $3.20 E-09$ \\
APS (order $=9)$ & Div & Div & $2.44 E-09$ & $4.20 E-10$ \\
APS (order $=11)$ & Div & Div & Div & $3.81 E-10$ \\
\hline
\end{tabular}

$$
\begin{gathered}
\left.+(\varepsilon v-2)\left(1+2 x_{2}\left(2+x_{2}\right)\right)\right] \cosh x_{2} \\
-\left[12(\varepsilon v-2)\left(1+x_{2}\right)\right. \\
\left.+\lambda^{2}(v-1)\left(9+x_{2}\left(9+2 x_{2}\left(3+x_{2}\right)\right)\right)\right] \\
\left.\left.\cdot \sinh x_{2}\right\}\right) \\
\cdot\left(48 \lambda^{2}(1-v)\right)^{-1} .
\end{gathered}
$$

Example 1. First, we consider a Reissner plate defined in $[-1,1]^{2}$ subjected to clamped boundary conditions, which are set according to the analytical solutions in (61).

The particular solutions of the present problem are solved by the DRM based on the APS [26], Chebyshev polynomials [25], and PMQ. Table 1 gives the RMSEs for the solutions obtained by the DRMs with different bases and N. Basically, the solutions obtained by the Chebyshev polynomials are the most accurate and the application is straightforward if the arbitrary loadings are given by functions. However, when the loadings are measured on some scattered points, a twostage method [31] is required which is relatively more difficult compared to the direct application of either APS or PMQ. In the table, the superior accuracy of the MQ over the first few orders of the APS can be observed. In addition, the accuracy improvement for the numerical solutions obtained by the high-order PMQ over the traditional MQ can also be observed. Overall, the best accuracy of the high-order PMQ is close to that of the high-order APS while the former RBF does not need augmented polynomials. In practice, it can be cumbersome to implement the particular solutions of the high-order augmented polynomials and thus the superiority of the PMQ over the APS becomes more relevant.

For both MQ and PMQ, the optimal shape parameters $c$ are searched as demonstrated in Figure 4. Basically, the numerical accuracy improves with increasing $c$ value. However, as $c$ is continuously made larger, the interpolation matrix becomes ill-conditioned [32]. Then, a typical error contour is given in Figure 5 for the solution obtained by the MFS-DRM based on the MQ. In the figure, the larger errors are on the boundary as expected.

Finally, the RMSE comparison for different numbers of nodes is given in Figure 6, in which higher resolution gives better accuracy. Overall, these results should have validated the polyharmonic multiquadric particular solutions of $u_{i}(r)$. 
TABLE 2: The RMSEs for Example 2.

\begin{tabular}{lcccc}
\hline & $N=6 \times 6$ & $N=8 \times 8$ & $N=10 \times 10$ & $N=12 \times 12$ \\
\hline Chebyshev & $5.36 E-06$ & $7.98 E-08$ & $4.43 E-08$ & $1.07 E-08$ \\
MQ & $5.51 E-05$ & $1.03 E-05$ & $1.42 E-06$ & $6.88 E-07$ \\
PMQ $(L=2)$ & $4.97 E-05$ & $5.07 E-06$ & $5.51 E-07$ & $1.15 E-07$ \\
PMQ $(L=4)$ & $3.12 E-05$ & $3.30 E-06$ & $1.64 E-07$ & $4.21 E-08$ \\
APS (order $=3)$ & $1.85 E-03$ & $4.77 E-04$ & $1.64 E-04$ & $6.88 E-05$ \\
APS (order $=5)$ & $1.11 E-04$ & $1.13 E-05$ & $2.27 E-06$ & $6.49 E-07$ \\
APS (order $=7)$ & Div & $4.20 E-06$ & $7.06 E-07$ & $1.63 E-07$ \\
APS (order $=9)$ & Div & Div & $5.66 E-08$ & $1.20 E-08$ \\
APS (order $=11)$ & Div & Div & Div & $1.08 E-08$ \\
\hline
\end{tabular}

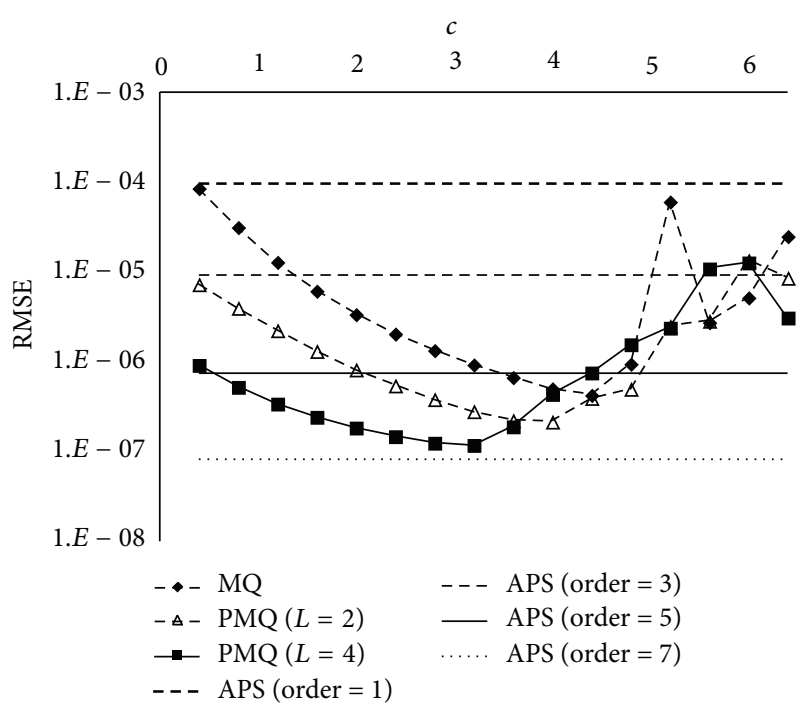

(a)

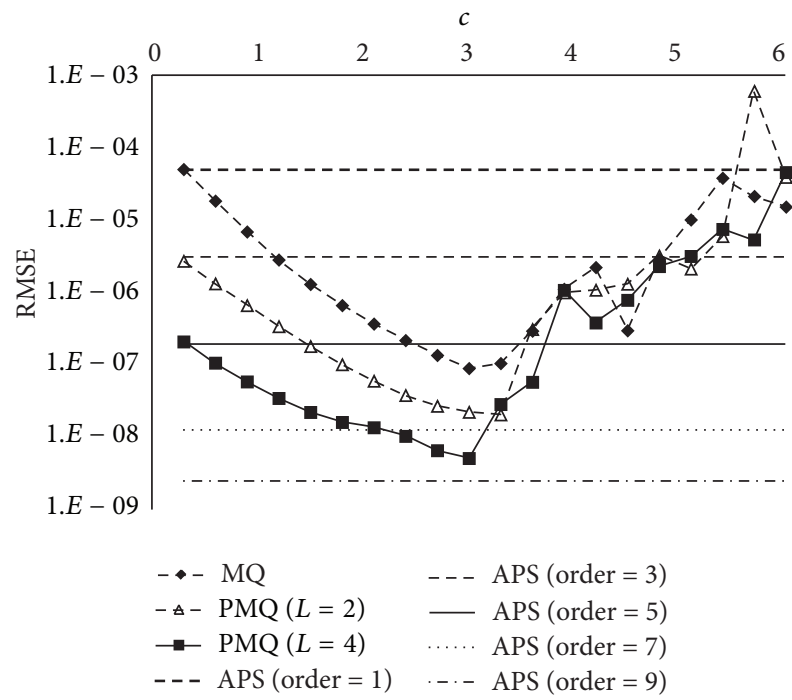

(b)

Figure 4: Optimal shape parameter searches of Example 1 for (a) $N=8 \times 8$ and (b) $N=10 \times 10$.

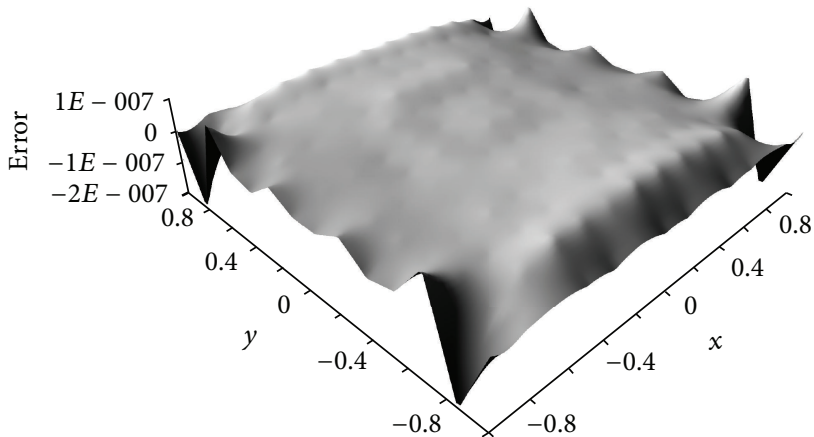

Figure 5: Error contour for Example 1 with $N=10 \times 10$ and $c=3$.

Example 2. Then, we consider a similar Mindlin plate problem by changing the boundary conditions to the free boundary condition on one side. Table 2 addresses the RMSEs for the numerical results in this example. The results are very similar except that the errors are slightly larger due to the numerical approximations on the free boundary condition.

In addition, the optimal shape parameter searches and the error comparison for different numbers of nodes are given in Figures 7 and 8, respectively. The results are similar to the previous example. Therefore, the polyharmonic multiquadric particular solutions of $Q_{\alpha}(r)$ and $\Upsilon_{\alpha \beta}(r)$ are also validated.

\section{Discussions}

In this paper, analytical particular solutions of the polyharmonic multiquadrics associated with Reissner and Mindlin thick plates in bending are derived. In the derivations, the coupled system of the three second-order governing equations is transformed into a single sixth-order product operator of biharmonic and Helmholtz operators. Then, a method is introduced to eliminate the Helmholtz operator, which enables the use of the polyharmonic multiquadric. Numerical results are performed to validate the derived particular solutions and to demonstrate the superiority of the polyharmonic multiquadrics over the traditional multiquadrics and the augmented polynomial splines. 


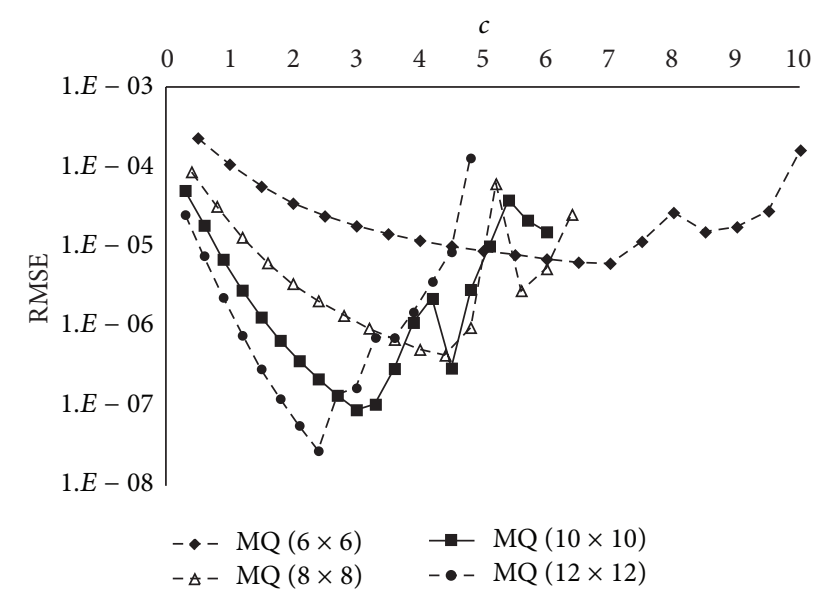

FIGURE 6: RMSE comparisons of Example 1 for different numbers of nodes.

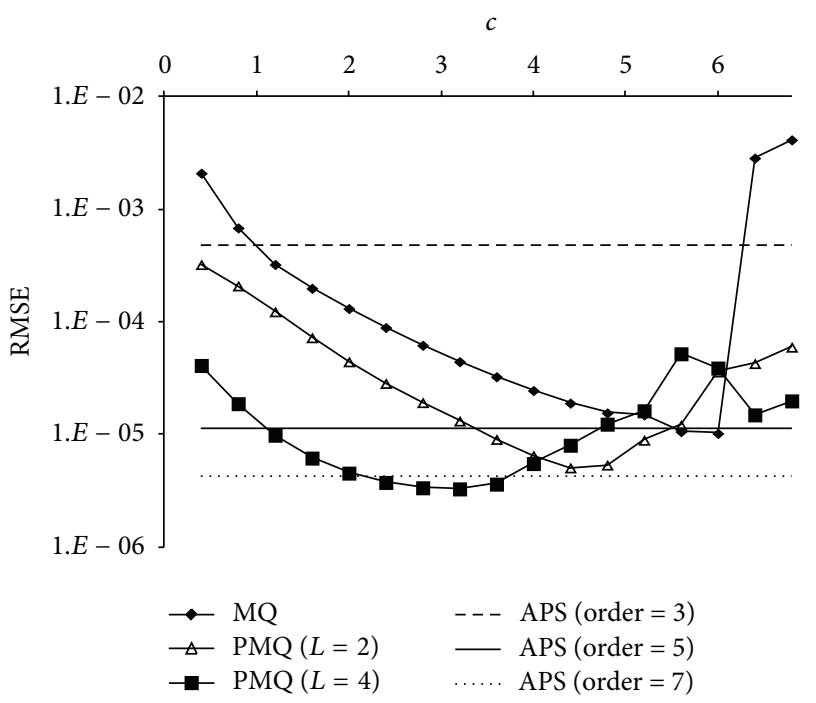

(a)

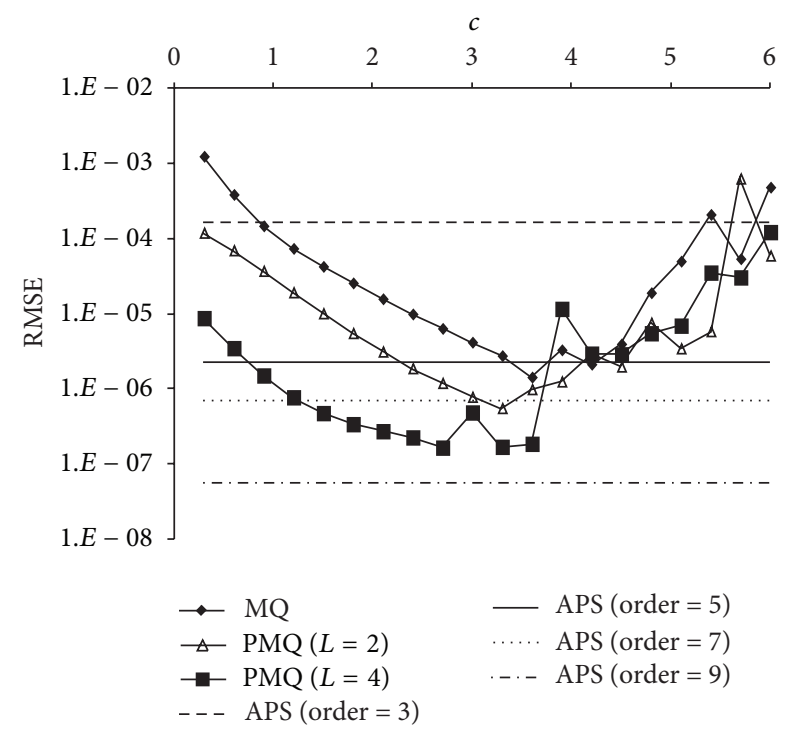

(b)

Figure 7: Optimal shape parameter searches of Example 2 for (a) $N=8 \times 8$ and (b) $N=10 \times 10$.

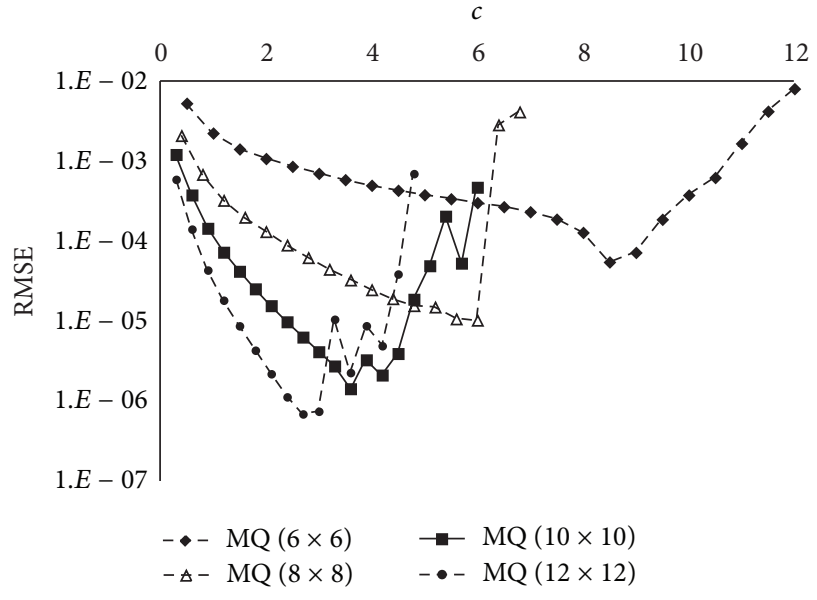

FIGURE 8: RMSE comparisons of Example 2 for different numbers of nodes.

\section{Conflict of Interests}

The author declares that there is no conflict of interests regarding the publication of this paper.

\section{Acknowledgment}

The National Science Council of Taiwan is gratefully acknowledged for providing financial support to carry out the present work under Grant no. MOST 103-2221-E-022-017MY2.

\section{References}

[1] A. H.-D. Cheng and D. T. Cheng, "Heritage and early history of the boundary element method," Engineering Analysis with Boundary Elements, vol. 29, no. 3, pp. 268-302, 2005.

[2] G. Fairweather and A. Karageorghis, "The method of fundamental solutions for elliptic boundary value problems," Advances in Computational Mathematics, vol. 9, no. 1-2, pp. 6995, 1998.

[3] M. A. Golberg and C. S. Chen, Eds., The Method of Fundamental Solutions for Potential, Helmholtz and Diffusion Problems, Computational Mechanics Publications, Southampton, UK, 1999.

[4] C.-S. Liu, "A highly accurate solver for the mixed-boundary potential problem and singular problem in arbitrary plane domain," CMES: Computer Modeling in Engineering and Sciences, vol. 20, no. 2, pp. 111-122, 2007.

[5] C.-S. Liu, "A highly accurate MCTM for direct and inverse problems of biharmonic equation in arbitrary plane domains," CMES: Computer Modeling in Engineering and Sciences, vol. 30, no. 2, pp. 65-75, 2008.

[6] P. W. Partridge, C. A. Brebbia, and L. C. Wrobel, The Dual Reciprocity Boundary Element Method, Computational Mechanics, Southampton, UK, 1992.

[7] D. Nardini and C. A. Brebbia, "A new approach to free vibration analysis using boundary elements," Applied Mathematical Modelling, vol. 7, no. 3, pp. 157-162, 1983.

[8] M. A. Golberg and C. S. Chen, "The theory of radial basis functions applied to the bem for inhomogeneous partial differential 
equations," Boundary Elements Communications, vol. 5, pp. 5761, 1994.

[9] S. R. Karur and P. A. Ramachandran, "Radial basis function approximation in the dual reciprocity method," Mathematical and Computer Modelling, vol. 20, no. 7, pp. 59-70, 1994.

[10] R. L. Hardy, "Multiquadric equations of topography and other irregular surfaces," Journal of Geophysical Research, vol. 76, no. 8, pp. 1905-1915, 1971.

[11] J. Duchon, "Splines minimizing rotation-invariant semi-norms in sobolev spaces," in Constructive Theory of Functions of Several Variables, vol. 571 of Lecture Notes in Mathematics, pp. 85-100, Springer, Berlin, Germany, 1977.

[12] M. A. Golberg, "Numerical evaluation of particular solutions in the BEM-a review," Boundary Elements Communications, vol. 6, no. 3, pp. 99-106, 1995.

[13] M. A. Golberg, "The method of fundamental solutions for Poisson's equation," Engineering Analysis with Boundary Elements, vol. 16, no. 3, pp. 205-213, 1995.

[14] C. S. Chen, "The method of fundamental solutions for nonlinear thermal explosions," Communications in Numerical Methods in Engineering, vol. 11, no. 8, pp. 675-681, 1995.

[15] M. A. Golberg, C. S. Chen, and S. R. Karur, "Improved multiquadric approximation for partial differential equations," Engineering Analysis with Boundary Elements, vol. 18, no. 1, pp. 9-17, 1996.

[16] C.-C. Tsai, "Analytical particular solutions of multiquadrics associated with polyharmonic operators," Mathematical Problems in Engineering, vol. 2013, Article ID 613082, 11 pages, 2013.

[17] M. F. Samaan and Y. F. Rashed, "BEM for transient 2D elastodynamics using multiquadric functions," International Journal of Solids and Structures, vol. 44, no. 25-26, pp. 8517-8531, 2007.

[18] M. F. Samaan and Y. F. Rashed, "Free vibration multiquadric boundary elements applied to plane elasticity," Applied Mathematical Modelling, vol. 33, no. 5, pp. 2421-2432, 2009.

[19] C.-C. Tsai and T.-W. Hsu, "Multiquadric and Chebyshev approximation to three-dimensional thermoelasticity with arbitrary body forces," Engineering Analysis with Boundary Elements, vol. 37, no. 10, pp. 1259-1266, 2013.

[20] A. H.-D. Cheng, C. S. Chen, M. A. Golberg, and Y. F. Rashed, "BEM for theomoelasticity and elasticity with body force-a revisit," Engineering Analysis with Boundary Elements, vol. 25, no. 4-5, pp. 377-387, 2001.

[21] G. Yao, The method of approximate particular solutions for solving partial differential equations [Doctor of Philosophy], Department of Mathematics, The University of Southern Mississippi, 2010.

[22] R. D. Mindlin, "Influence of rotary inertia and shear on flexural motions of isotropic, elastic plates," Journal of Applied Mechanics, vol. 18, pp. 336-343, 1951.

[23] P. H. Wen, M. H. Aliabadi, and A. Young, "Transformation of domain integrals to boundary integrals in BEM analysis of shear deformable plate bending problems," Computational Mechanics, vol. 24, no. 4, pp. 304-309, 1999.

[24] C.-C. Tsai and E. M. Wu, "Analytical particular solutions of augmented polyharmonic spline associated with mindlin plate model," Numerical Methods for Partial Differential Equations, vol. 28, no. 6, pp. 1778-1793, 2012.

[25] C.-C. Tsai, "The particular solutions of chebyshev polynomials for reissner plates under arbitrary loadings," CMES: Computer Modeling in Engineering and Sciences, vol. 45, no. 3, pp. 249-271, 2009.
[26] C. C. Tsai, M. E. Quadir, H. H. Hwung, and T. W. Hsu, "Particular solution of polyharmonic spline associated with reissner plate problems," Journal of Mechanics, vol. 27, no. 4, pp. 493-501, 2011.

[27] E. Reissner, "The effect of transverse shear deformation on the bending of elastic plates," Journal of Applied Mechanics, vol. 12, pp. 69-76, 1945.

[28] E. Reissner, "On the theory of bending of elastic plates," Journal of Applied Mechanics, vol. 23, pp. 184-191, 1945.

[29] L. Hörmander, Linear Partial Differential Operators, Springer, Berlin, Germany, 1963.

[30] H. Wendland, "On the smoothness of positive definite and radial functions," Journal of Computational and Applied Mathematics, vol. 101, no. 1-2, pp. 177-188, 1999.

[31] S. Y. Reutskiy and C. S. Chen, "Approximation of multivariate functions and evaluation of particular solutions using Chebyshev polynomial and trigonometric basis functions," International Journal for Numerical Methods in Engineering, vol. 67, no. 13, pp. 1811-1829, 2006.

[32] R. Schaback, "Error estimates and condition numbers for radial basis function interpolation," Advances in Computational Mathematics, vol. 3, no. 3, pp. 251-264, 1995. 


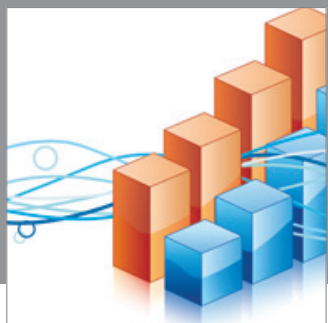

Advances in

Operations Research

mansans

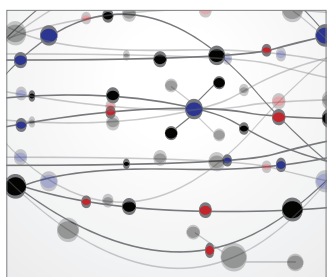

The Scientific World Journal
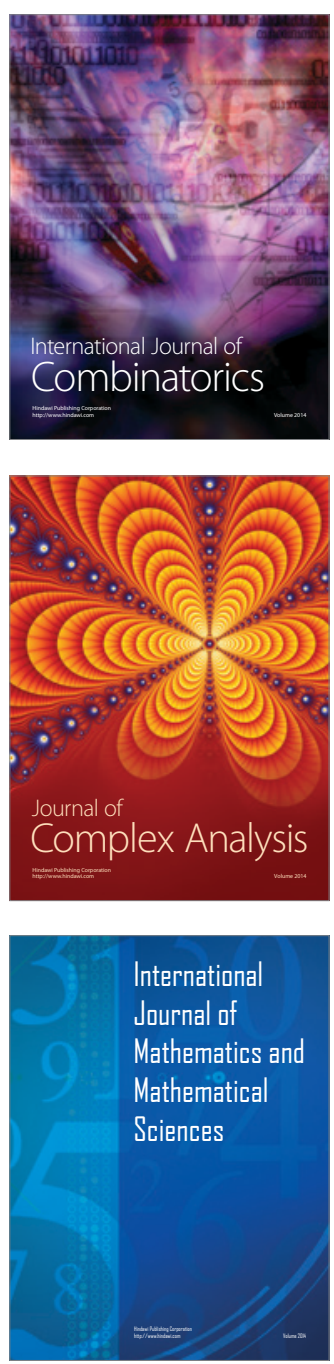
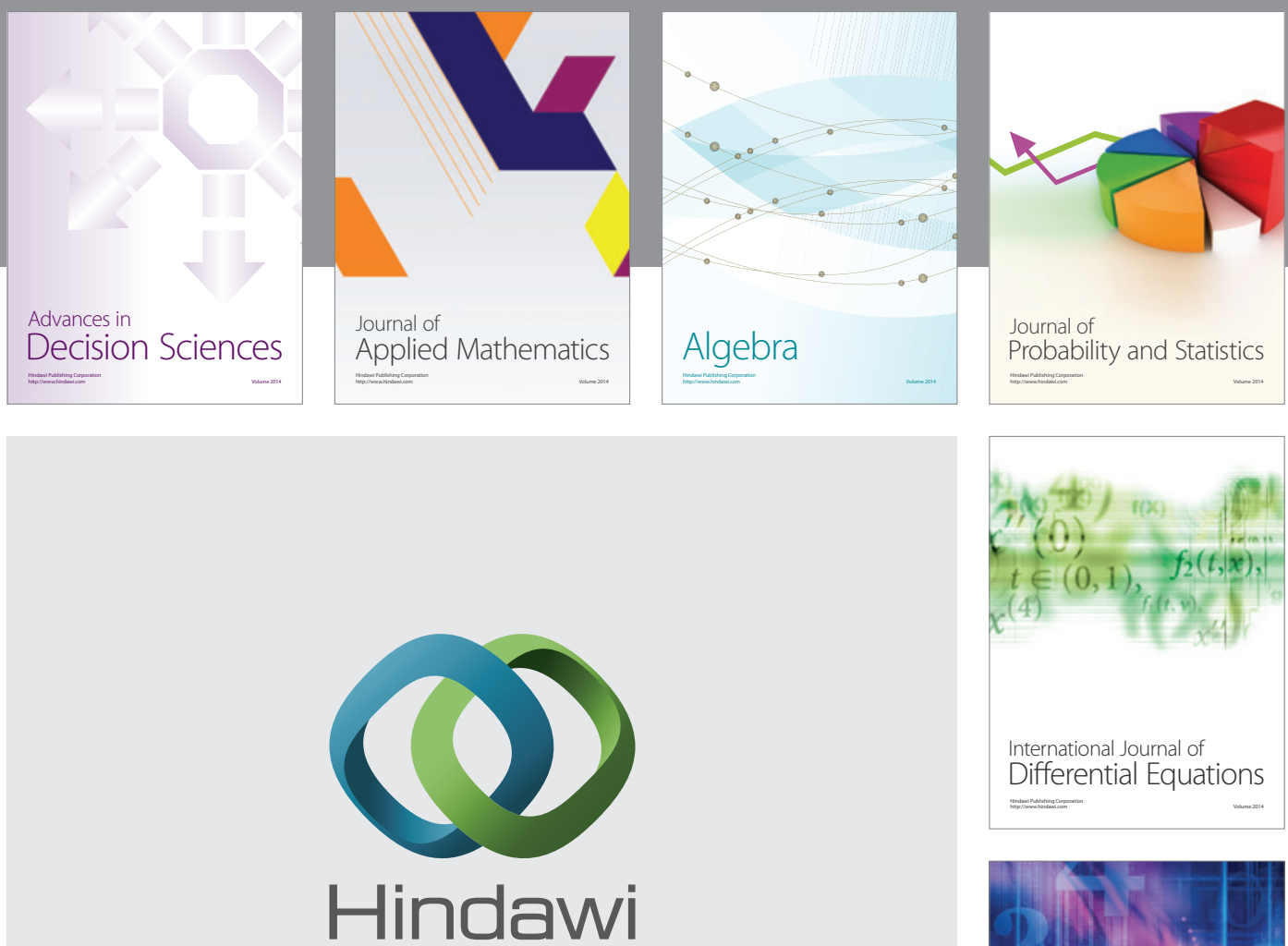

Submit your manuscripts at http://www.hindawi.com
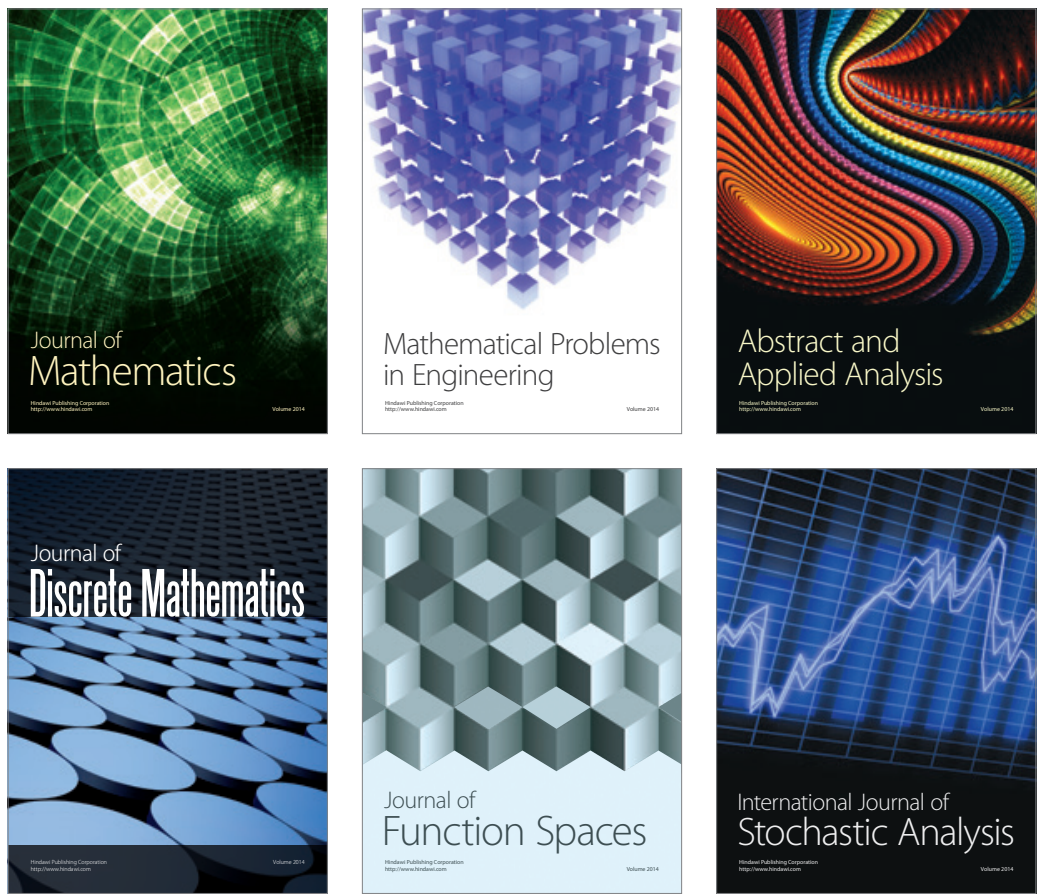

Journal of

Function Spaces

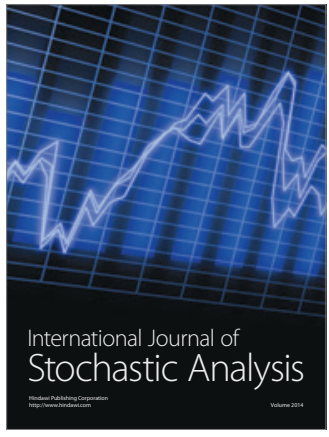

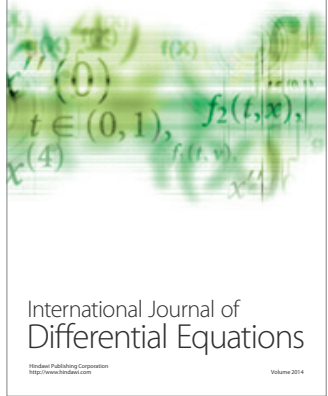
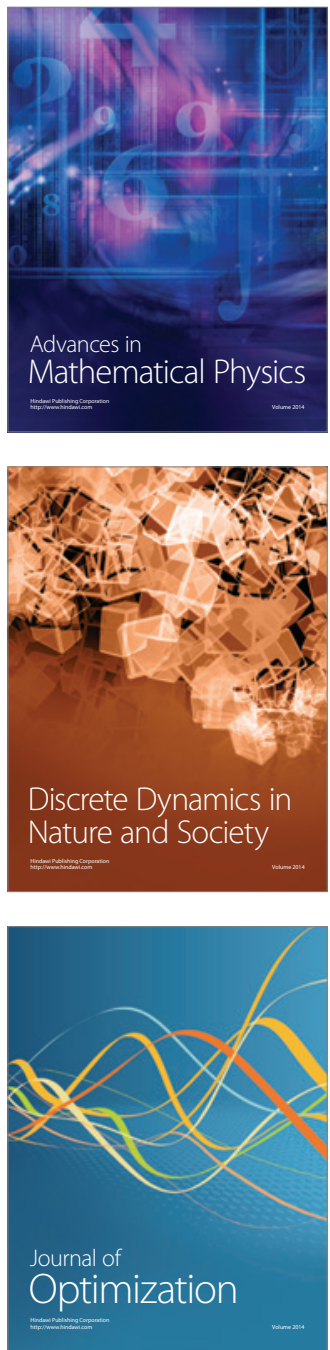